\title{
Measurement of double differential cross-section of light water at high temperature and pressure to generate $S(\alpha, \beta)$
}

\author{
Vaibhav Jaiswal ${ }^{1, \text { a }}$, Luiz Leal ${ }^{1}$, Wim Haeck ${ }^{1}$, Emmanuel Farhi $^{2}$, Yoann Calzavara ${ }^{2}$, Stéphane Rols ${ }^{2}$, Jacques Ollivier ${ }^{2}$, \\ Gilles Noguere ${ }^{3}$, Juan Pablo Scotta ${ }^{3}$, Valérie Vallet ${ }^{4}$, and Florent Réal ${ }^{4}$ \\ ${ }^{1}$ Institut de Radioprotection et de Sûreté Nucléaire (IRSN), PSN-EXP/SNC/LNR, Fontenay-aux-Roses, France \\ 2 Institut Laue-Langevin, CS-Group, Grenoble, France \\ 3 CEA, DEN, DER, Cadarache, Saint-Paul-les-Durance, France \\ ${ }^{4}$ Univ. Lille, CNRS, UMR 8523 - PhLAM - Physique des Lasers Atomes et Molécules, 59000 Lille, France
}

\begin{abstract}
A series of double differential inelastic scattering cross-section measurements were performed on light water at several temperatures and pressures using high resolution time-of-flight inelastic spectrometers, namely the IN4c and the IN6 at the Institut Laue-Langevin (ILL), Grenoble, France to investigate the impact of temperature and pressure on $\mathrm{S}(\mathbf{q}, \omega)$ and thus on the $\mathrm{S}(\alpha, \beta)$ thermal scattering kernel. The present work aims at extending previous measurements with light water at room temperature and pressure to more realistic operating conditions in connection with nuclear power reactors.
\end{abstract}

\section{Introduction}

Light water is the most widely used moderator in nuclear power reactors, namely the pressurized water reactors (PWRs). Reliable thermal neutron scattering cross-section data for light water is thus necessary for criticality safety studies and reactor physics applications. Neutron scattering cross-section data for several moderator materials are available in standard nuclear cross-section data libraries, represented by $\mathrm{S}(\alpha, \beta)$ thermal scattering kernel where $\alpha$ and $\beta$ stand for the unit-less momentum and energy transfers respectively.

$\mathrm{S}(\alpha, \beta)$ of light water in the evaluated nuclear data libraries are derived on the basis of physics models [1] describing neutron interaction at low energy including the effects of binding atoms/molecules on the system. The evaluation process used in these models is based on the experimental data measured in the 1960s with a low accuracy due to the limitations of the experimental and computational capabilities. Since then, much progress has been made in re-evaluating the thermal scattering crosssection data for light water by incorporating molecular dynamics simulations and available experimental data [2] but unfortunately new experimental data for light water especially at high temperature and pressure is very scarce and the new evaluations rely on these old experimental data $[3,4]$. In particular, there is a need for new experimental data for $\mathrm{S}(\alpha, \beta)$ of light water matching reactor normal operating conditions (Pressure $\sim 150$ bar and Temperature $\sim 600 \mathrm{~K})$ as $\mathrm{S}(\alpha, \beta)$ strongly depends on temperature. Using $\mathrm{S}(\alpha, \beta)$ at the appropriate temperature is necessary to study the temperature effects in reactor physics calculations since a slightly different temperature for the $\mathrm{S}(\alpha, \beta)$ table can result in a fundamentally different behavior of reactor parameters [5].

a e-mail: vaibhav.jaiswal@irsn.fr
The Institut de Radioprotection et de Sûreté Nucléaire (IRSN) which provides technical support for the French nuclear safety authority, is working on the improvement of thermal scattering cross-section data of light water for the last few years as part of the NAUSICAA collaboration. Improved experiments were performed on light water at ambient temperature and pressure at the Institut LaueLangevin (ILL), Grenoble, France in 2010 to generate $\mathrm{S}(\alpha, \beta)$ [6]. In this paper we present the results of the two consecutive inelastic thermal neutron scattering experiments on light water, performed with high resolution time-of-flight (TOF) spectrometers, namely the IN4c and the IN6 at ILL in December 2015 [7]. This way we have tried to extend the previous measurements beyond room temperature and pressure to more realistic operating conditions in connection with nuclear power reactors. Measurements were carried out for several thermodynamic states of light water. This paper investigates the impact of temperature and pressure on the thermal scattering crosssection data of light water especially the experimental double differential scattering cross-section. In addition, the data uncertainties can also be obtained from the experimental data which is under process.

\section{Theoretical framework}

Using the first Born approximation and Fermi pseudopotential [1], the double differential scattering crosssection of light water at a particular temperature $\mathrm{T}$ can be described as the number of neutrons which are scattered per unit time into the solid angle interval $\left[\Omega, \Omega^{\prime}=\Omega+d \Omega\right]$ and into the energy interval $\left[E, E^{\prime}=E+d E\right]$ which is expressed as:

$$
\frac{\partial^{2} \sigma_{T}}{\partial \Omega \partial E^{\prime}}\left(E \rightarrow E^{\prime}, \Omega \rightarrow \Omega^{\prime}\right)=\frac{\sigma_{\mathrm{b}}}{4 \pi K_{B} T} \sqrt{\frac{E^{\prime}}{E}} e \frac{\beta}{2} S(\alpha, \beta)
$$

(c) The Authors, published by EDP Sciences. This is an Open Access article distributed under the terms of the Creative Commons Attribution License 4.0 (http://creativecommons.org/licenses/by/4.0/). 
where $E$ and $E^{\prime}$ are the incident and secondary neutron energies in the laboratory system, $\Omega$ is the scattering angle in the laboratory system, $\sigma_{b}$ is the bound scattering crosssection for light water, $\mathrm{K}_{B}$ is the Boltzmann constant and $\mathrm{S}(\alpha, \beta)$ is the $\beta$-symmetric form of the thermal scattering law with:

$$
\alpha=\frac{E^{\prime}+E-2 \sqrt{E E^{\prime}} \cos \theta}{A K_{B} T} \text { and } \beta=\frac{E^{\prime}-E}{K_{B} T}
$$

where $\cos \theta$ is the $\operatorname{cosine}$ of the scattering angle in the laboratory system and $\mathrm{A}=M / m$ with $M$ being the mass of the water molecule and $m$ the neutron mass.

$\mathrm{S}(\alpha, \beta)$ is analogous to the dynamic structure factor $\mathrm{S}(\mathbf{q}, \omega)$ which describes the microscopic dynamics of a scattering system, where $\mathbf{q}$ is the neutron momentum transfer and is the neutron energy transfer in units of $\hbar$. $\mathrm{S}(\alpha, \beta)$ can be computed from symmetric $\mathrm{S}(\mathbf{q}, \omega)$ as follows:

$$
S(\alpha, \beta)=K_{B} T S^{s y m}(q, \omega)
$$

with the parameters:

$$
\alpha=\frac{\mathrm{q}^{2} \hbar^{2}}{2 \mathrm{M} K_{B} \mathrm{~T}} \quad \text { and } \quad \beta=-\frac{\hbar \omega}{K_{B} T}
$$

The quantity of interest in neutron scattering experiments with thermal neutrons is this dynamic structure factor $\mathbf{S}(\mathbf{q}, \omega)$ which is related to the double differential scattering cross-section by Eq. (1). $\mathbf{S}(\mathbf{q}, \omega)$ for light water can be directly measured by performing TOF inelastic thermal neutron scattering experiment in order to avoid approximations [1] and thus to possibly enhance the reliability of $\mathrm{S}(\alpha, \beta)$ and the accuracy of the thermal scattering cross-sections.

\section{Time-of-flight experiment [TOF]}

In order to obtain the dynamic structure factor $S(\mathbf{q}, \omega)$ we have carried out TOF experiments at ILL, that provides a powerful source of neutrons produced by a high flux reactor (HFR). For our purpose we chose two TOF spectrometers namely the IN4c and the IN6. Instruments at ILL are identified by the instrument's symbols which correspond to experimental techniques i.e., IN stands for Inelastic neutron scattering. The idea behind choosing two different spectrometers is to cover the entire momentum and energy transfer ranges within the experimental limitations of each instrument.

\subsection{IN4C}

IN4c is a direct geometry TOF spectrometer which works in the thermal neutron energy range between 10 to $100 \mathrm{meV}$, offering a high flux $\sim 5 \times 10^{5} \mathrm{n} / \mathrm{cm}^{2} / \mathrm{s}$ at the sample position [8]. A schematic representation of the instrument is given in Fig. 1. This instrument has efficient background suppression by two background choppers that eliminate background noise in the spectra [9]. A suitable energy is selected from the thermal neutron spectrum with a $\mathrm{Cu}$ (III) crystal monochromator. Good counting rate is ensured by a large detector bank with ${ }^{3} \mathrm{He}$ tubes covering scattering angles up to $120^{\circ}$.

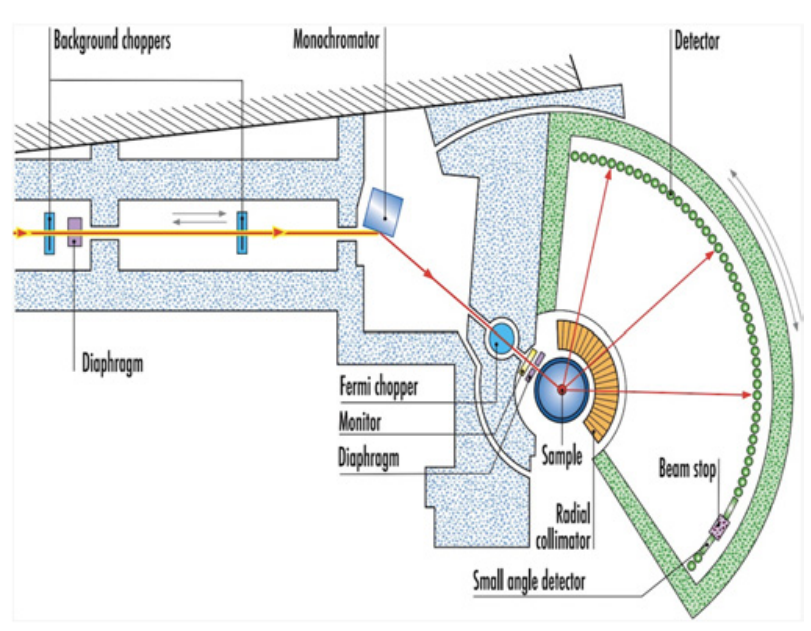

Figure 1. Schematic representation of the IN4c TOF spectrometer at the Institut Laue-Langevin [8].

For our measurements with IN4c, wavelength of the incident neutron was chosen as $2.41 \AA$. This wavelength corresponds to a neutron energy of $14.2 \mathrm{meV}$. Speed ratio of background/Fermi chopper was chosen as 1:2 to minimize frame overlap and to extend the time range for data acquisition. Studying the FWHM of the elastic peak of a vanadium sample at this wavelength gives the energy resolution of about $4 \%$. The sample environment is designed to accommodate standard cryo-furnace which can maintain high pressure and temperature. Secondary flight-path which is $300 \mathrm{~cm}$ is in vacuum to avoid parasitic scattering of the transmitted neutrons.

\subsection{IN6}

IN6 is a direct geometry TOF spectrometer designed for quasi-elastic and inelastic scattering experiments [8]. A schematic diagram of the IN6 TOF spectrometer is given in Fig. 2. This instrument comprises of three vertically focusing pyrolytic graphite monochromatic crystals, which can provide incident wavelengths in the range of 4 to $6 \AA$ by choosing the appropriate one. Fermi chopper is used for the time focusing and the distance between Fermi chopper and the sample is $38 \mathrm{~cm}$ while the sample to detector distance is $248 \mathrm{~cm}$. The flux that can be achieved at the sample position is $\sim 8.9 \times 10^{4} \mathrm{n} / \mathrm{cm}^{2} / \mathrm{s}$ [8]. There are 337 elliptical ${ }^{3} \mathrm{He}$ detectors that cover an angular range of $10^{\circ}-115^{\circ}$. Wavelength of the incident neutron for measurements with IN6 was chosen as $5.1 \AA$. This wavelength corresponds to a neutron energy of $3.15 \mathrm{meV}$. Speed ratio was chosen as 1:1 with extended time range for data acquisition and to minimize the overlap. Elastic energy resolution obtained by choosing this incident neutron wavelength is about $2.2 \%$.

\subsection{Data acquisition, reduction and analysis}

In order to utilize the maximum of the beam time allotted at the ILL facility for both the IN4c and IN6 spectrometers, we chose several thermodynamic states of light water (pressure and temperature combinations) as shown in Fig. 3, to cover the liquid phase of light water respecting the constraint imposed by the cryostat and the pressure cell (maximum temperature and pressure). 


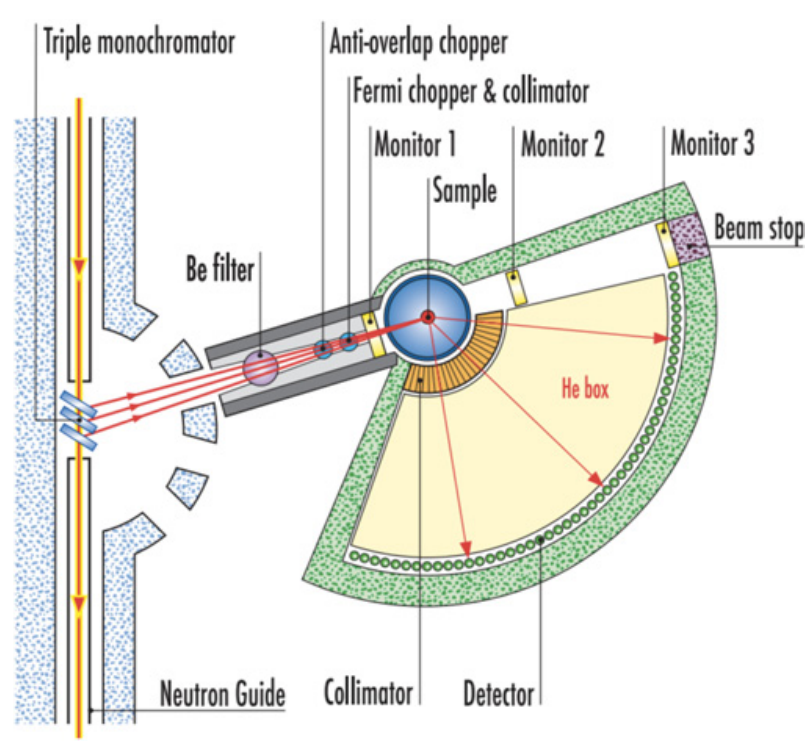

Figure 2. Schematic representation of the IN6 TOF spectrometer at the Institut Laue-Langevin [8].

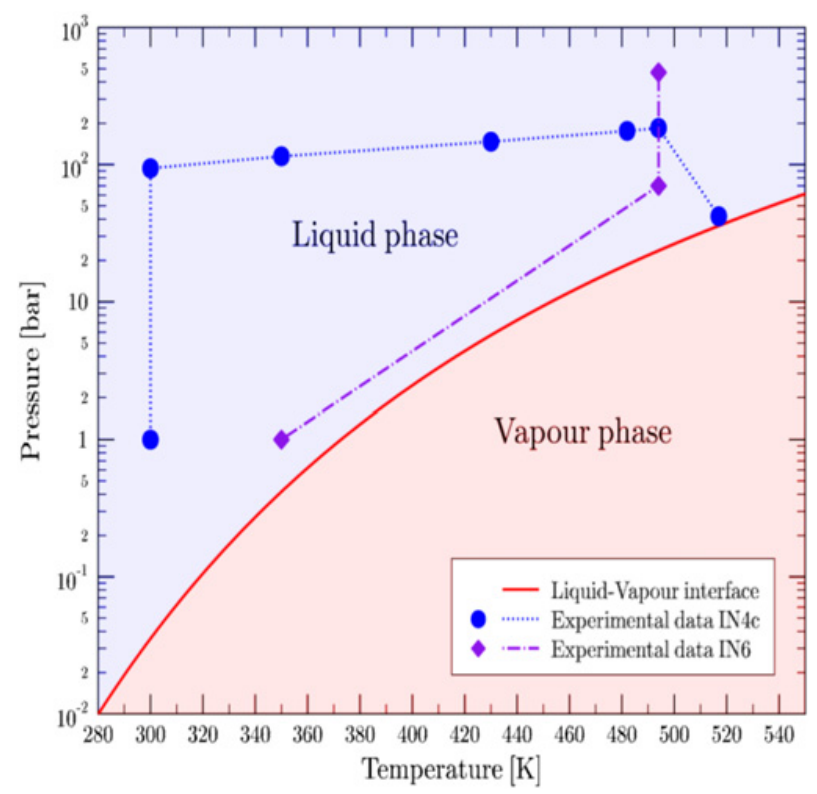

Figure 3. Thermodynamic states (experimental conditions) of light water for the data measurement.

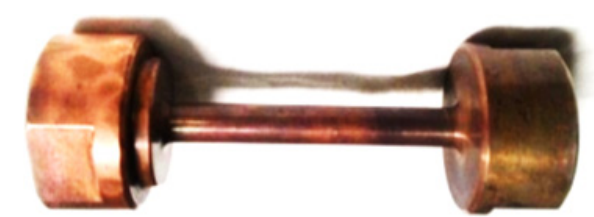

Figure 4. CuBe pressure cell (sample holder).

The cryogenics team at ILL developed a special cylindrical pressure cell (sample holder) of $\mathrm{CuBe}(\Phi=0.6 \mathrm{~cm}$ interior, $0.8 \mathrm{~cm}$ exterior; height $=7 \mathrm{~cm}$ ) as shown in Fig. 4, which can withstand high temperature and pressure and can easily fit in a cryo-furnace. High purity water sample was inserted in this pressure cell for the TOF measurements with both the spectrometer.
Table 1. Experimental conditions with the IN4c and the IN6 TOF spectrometers.

\begin{tabular}{|c|c|c|c|}
\hline \multicolumn{4}{|c|}{ Experimental conditions with IN4c \& IN6 } \\
\hline $\begin{array}{c}\text { Time-of- } \\
\text { flight } \\
\text { spectrometer }\end{array}$ & $\begin{array}{c}\text { Temperature } \\
\text { (K) }\end{array}$ & $\begin{array}{c}\text { Pressure } \\
\text { (bar) }\end{array}$ & $\begin{array}{c}\text { Data } \\
\text { Acquisiti- } \\
\text { on time } \\
\text { (hours) }\end{array}$ \\
\hline \multirow{7}{*}{$\begin{array}{c}\text { IN4c } \\
\lambda=2.4 \AA ; \\
\mathrm{E}=14.2 \mathrm{meV}\end{array}$} & 300 & 1 & 2.5 \\
\hline & 300 & $\begin{array}{c}88-100 \\
(94)\end{array}$ & 2 \\
\hline & 350 & 115 & 2 \\
\hline & $\begin{array}{c}392-466 \\
(430)\end{array}$ & $\begin{array}{c}128-165 \\
(147)\end{array}$ & $\sim 1$ \\
\hline & $\begin{array}{c}479-485 \\
(482)\end{array}$ & $\begin{array}{c}172-180 \\
(176)\end{array}$ & $\sim 1$ \\
\hline & $\begin{array}{c}490-497 \\
(494)\end{array}$ & 185 & 10.5 \\
\hline & 517 & 42 & 3 \\
\hline \multirow{3}{*}{$\begin{array}{c}\text { IN6 } \\
\lambda=5.1 \AA ; \\
\mathrm{E}=3.15 \mathrm{meV}\end{array}$} & 350 & 1 & 4 \\
\hline & 494 & 70 & 9 \\
\hline & 494 & $\begin{array}{c}340-600 \\
(470)\end{array}$ & 4 \\
\hline
\end{tabular}

Table 1 represents the experimental conditions for which measurements were carried out. We saw some fluctuations in the temperature and pressure during the measurements for different experimental runs. The numbers in bracket represents the average temperature and pressure for which the data analysis is carried out.

Measurements were also done for the empty pressure cell (without light water) with both spectrometers to subtract the contribution of the $\mathrm{CuBe}$ from the measurements of light water. Similar approach was applied to a vanadium (cylindrical) reference to normalize the data with its elastic peak. A detailed data reduction of the raw data [7] was done using LAMP [10] and iFit [11] software to normalize the data with the counting time and vanadium reference, correct for the detector efficiency of each spectrometer and subtract the empty pressure cell. Multiple scattering is not taken into account in the present analysis. $\mathrm{S}(\mathbf{q}, \omega)$ was obtained for each experimental conditions and was then transformed into $\mathrm{S}(\alpha, \beta)$ using Eq. (3) in order to calculate the experimental double differential thermal neutron scattering cross-section using Eq. (1).

\section{Results}

The experimental double differential thermal neutron scattering cross-section is calculated by interpolating $\mathrm{S}(\alpha, \beta)$ for the required final energies using Eq. (1). Figure 5 and Fig. 6 represent the experimental double differential scattering cross-section of light water obtained from the measurements performed with the IN4c and the IN6 TOF spectrometers for an incident neutron of energy 


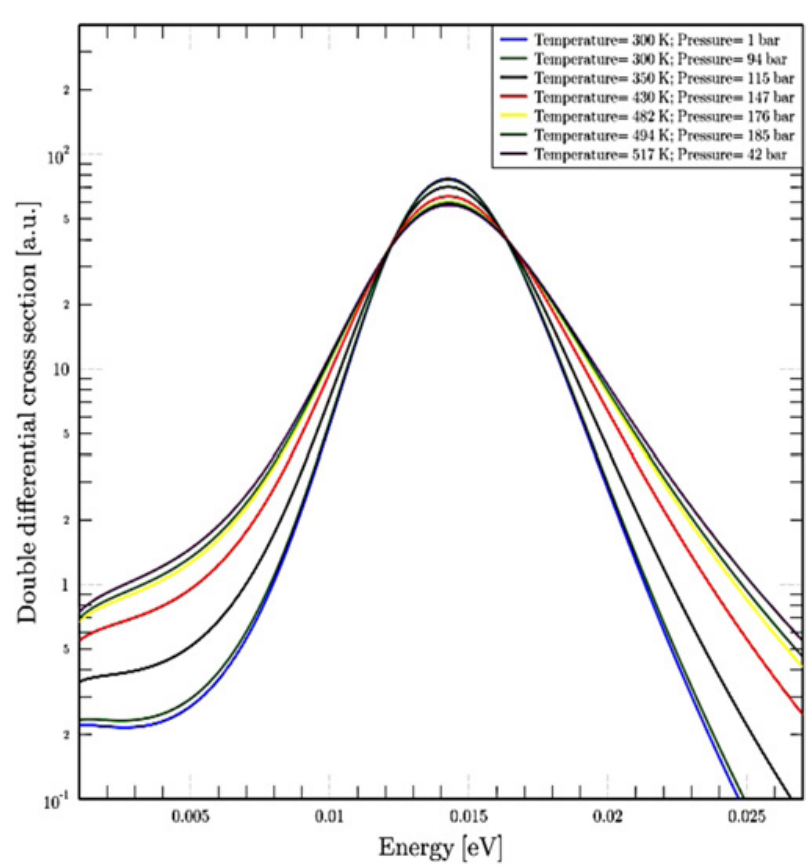

Figure 5. Experimental double differential scattering crosssection of light water measured at the IN4c for an incident energy $14.20 \mathrm{meV}$ and scattering angle $15^{\circ}$.

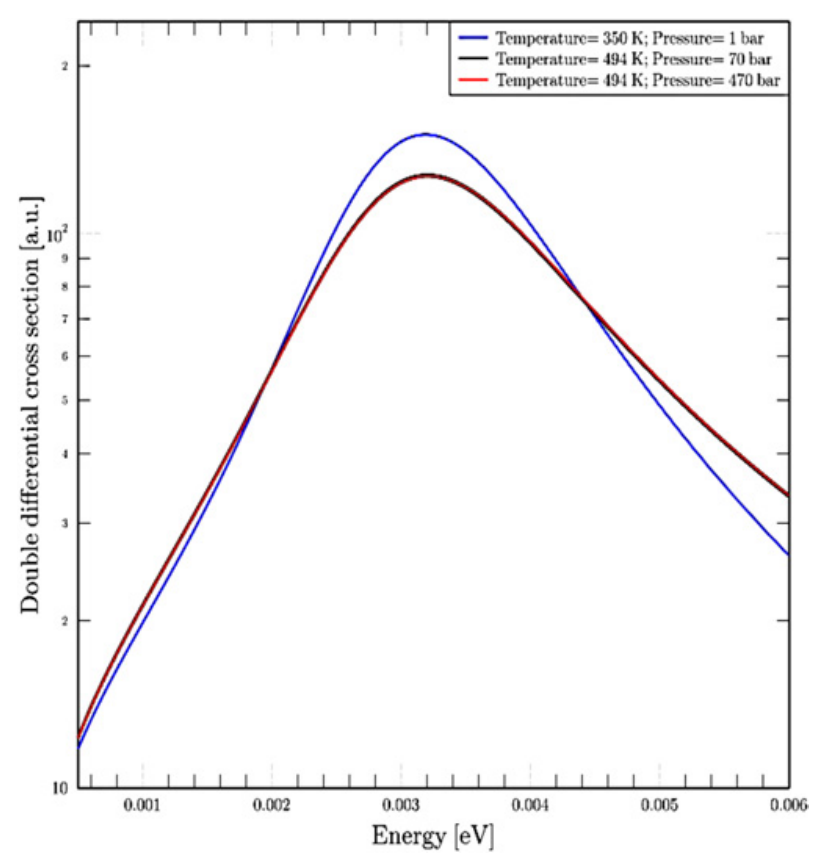

Figure 6. Experimental double differential scattering crosssection of light water measured at the IN6 for an incident energy $3.15 \mathrm{meV}$ and scattering angle $15^{\circ}$.

$14.20 \mathrm{meV}$ and $3.15 \mathrm{meV}$ respectively. Scattering angle is chosen arbitrarily as $15^{\circ}$ for both the data sets.
The goal of this work was to investigate the impact of temperature and pressure on the $\mathrm{S}(\alpha, \beta)$ thermal scattering kernel of light water by analyzing the experimental double differential thermal neutron scattering crosssection. Figure 5 and Fig. 6 shows some interesting results about the experimentally measured double differential scattering cross-section at different temperatures and pressure. The experimental double differential cross section decreases with the increasing temperature but is negligibly effected by the changing pressure.

\section{Conclusions}

Inelastic double differential scattering cross-section measurements were performed on light water using high resolution TOF spectrometers, namely the IN4c and the IN6 at the Institut Laue-Langevin, Grenoble, France. Several temperatures and pressures, matching the normal operating conditions of nuclear power reactors were investigated and their impact on the experimental double differential thermal neutron scattering cross-section was studied. This experimental analysis indicates that there is a weak dependence of pressure and a strong dependence of temperature on the experimental double differential crosssection of light water.

\section{References}

[1] R.E. MacFarlane, LANL Technical Report, "New Thermal Neutron Scattering Files for ENDF/B-VI, Release 2", LA-12639-MS (ENDF-356) (1994)

[2] J.I. Marquez Damian et al, Ann. Nucl. Ener. 65, 280 (2014)

[3] K. Heinloth, Z. Phys. 163, 218 (1961)

[4] K.N. Zaitsev, V.N. Petrov, S.P. Kuztnesov, O.A. Langer, I.V. Meshkov, A.D. Perekrestenko, Sov. At. Energy 70, 238 (1991)

[5] W. Haeck, N. Leclaire, Thermal scattering data and criticality safety, International Conference on the Physics of Reactors, Interlaken, Switzerland, September 14-19 (2008)

[6] E. Farhi et al., J. Nucl. Sci. Technol. 52(6), 844-856 (2015)

[7] E. Farhi et al, Measurement of light and heavy water thermal cross sections for neutron transport codes, up to $350 \mathrm{C}$ and 150 bar, Institut Laue-Langevin (ILL) doi:10.5291/ILL-DATA.1-04-90 (2015)

[8] Time-of-flight spectrometers IN4 and IN6, Guide to the Neutron Research Facilities, The Yellow Book, Institut Laue-Langevin, Grenoble, France (2008)

[9] G. Cicognani, The thermal neutron time-of-flight spectrometer IN4C, Physica B 276-278, 83-84 (2000)

[10] D. Richard, M. Ferrand, G.J. Kearley, J Neutron Res. 4, 33-39 (1996)

[11] E. Farhi, Y. Debab, P. Willendrup, J Neutron Res. 17, 5-18 (2013) 